\title{
A RECENT STUDY ON GEOMORPHOLOGY, CHARACTERISTICES AND CLASSIFICATION OF SOILS IN EL-FAYOUM GOVERNORATE, EGYPT
}

\author{
M.S. Amira ${ }^{(1)}$, A.H. El-Nahry ${ }^{(2)}$, F.E. Abu Agwa ${ }^{(1)}$ and E. A. A. Abdelsamie (2) \\ (1) Soil Sci. Dept., Fac. Agric. Menoufia univ . \\ (2) National Authority for Remote Sensing and Space Sciences (NARSS)
}

Received: May 9, 2021

Accepted: May 25, 2021

ABSTRACT: The current work aims to make a recent study on the geomorphology, characteristics and classification of soils in El-Fayoum governorate, Egypt. The investigated area is located to the west of the Nile at $90 \mathrm{~km}$ southwest of Cairo. It occupies $2138.53 \mathrm{~km}^{2}$. The agricultural land represents $1252.4 \mathrm{~km}^{2}$, meanwhile, the urban areas occupy $540.86 \mathrm{~km}^{2}$. The Multi Images produced by Remote Sensing and GIS technique were used to identify the geomorphological features of the area. A reconnaissance survey followed by detailed one was done to verify the information resulted from satellite images. Five main landforms with some sub-mains were recognized in the studied area namely: Recent river terraces (high, moderate and low), Basins (overflow, and decantation), Old river terraces (high, moderate high, moderate and low), Former lake bed, Water bodies (fish ponds and Lake Qaroun). Thirty soil profiles were chosen representing these landforms and morphologically described. Soil samples were collected for physiochemical analyses.

The studied area has almost flat topography with very deep soil materials. Some soils that having moderately high-water table levels, are moderately deep and imperfectly drained. Most of the studied soils have clay loam texture grade and some have loam to sandy loam ones. All soils have slightly alkaline reaction and most of them are slightlysaline with moderate to high sodicity effect especially the areas with high water table and/or adjacent to Lake Qaroun. These soils are moderately calcareous poor in organic matter and gypsum contents.

Most of the studied soils haven't any clear diagnostic horizons and classified according to Soil Survey Staff (2014) under Entisols. Some of them that have Natric and/or Calcic horizons are classified under Aridisols orders up to sub-great group level.

Key words: RS, GIS, geomorphic units, landforms, soil characteristics, soil classification.

\section{INTRODUCTION}

The challenge of agriculture in the $21^{\text {st }}$ century especially in Egypt requires an integrated and systematic approach. This approach must address sustainable use and management of natural resources through the development and adoption of farming technology and management practices that will ensure food security and agricultural livelihoods. The need for food for the population increasing in Egypt is threatening natural resources as people strive to get the most out of land already in production or push into virgin territory for new agricultural land. The damage is increasingly evident: the loss of arable lands owing to erosion, salinity, desertification, urban spread, water scarcity; global warming and climate change as well as threats to biodiversity (Hegazi et al., 2005).

The agricultural area in Egypt is composed of the Nile Valley and Delta, which considered the main contributor to 
food production, trading activities, and the national economy. Sustainable agricultural development requires a systematic effort towards the planning of land use activities in the appropriate way, apart from several other institutional and policy programmer initiatives. Heavy population pressure and the related increased competition from different types of land users have emphasized the need for more effective land-use planning and management. The Egyptian government adopted policies of selfsufficiency in food production, e.g., extension of cultivated land and maximization of production of the existing agricultural land. The principal purpose was and still is to overcome Egypt's overwhelmingly unfavorable population to agricultural land ratio. Such yearly progressive increase requires paying considerable attention to conserve our limited land resources to optimize our agricultural productivity per unit area and to maximize the agricultural reclaimed lands through a series of projects to develop new lands in the desert. Therefore, the Egyptian government places a high priority on exploring the natural resources in the Western Desert (Hegazi et al., 2005).

The identification

of geomorphological characteristics is from the important initial stages for studying any areas (Dawoud et al., 2005). Remote sensing (RS) is now recognized as an important tool in monitoring and managing natural resources (Lillesand and Kiefer, 2007). They added that RS technique is one of the important methods used for soil survey, mapping, and environmental investigation. ESRI (2003) stated that a geographic information system (GIS) is a system for the management, analysis, and displaying of geographic information, which is represented by a series of geographic datasets that model geography using simple, generic data structures.

The aim of the present work was to make a recent study on the geomorphology, physicochemical characteristics and classification of the soils in El-Fayoum governorate. The integration of remote sensing (RS) and geographic information system (GIS) techniques was used to perform all results and produce the spatial digital maps of this work.

\section{MATERIALS AND METHODS}

\section{Study area}

El Fayoum depression is located to the west of the river Nile at $90 \mathrm{~km}$ southwest of Cairo city. It is located between latitudes $29^{\circ} 05$ to $29^{\circ} 36 \mathrm{~N}$, and longitudes $30^{\circ} 20^{\prime}$ to $31^{\circ} 05^{\prime} \mathrm{E}$ (Fig, 1). It is geographically bounded by the Western Desert and the Nile Basin. It occupies 2138.53 km2 (509173.81 fed.). The agricultural land represents 1252.4 km2 (298190.48fed), meanwhile, the urban areas occupy 540.86 km2 (128776.19 fed).

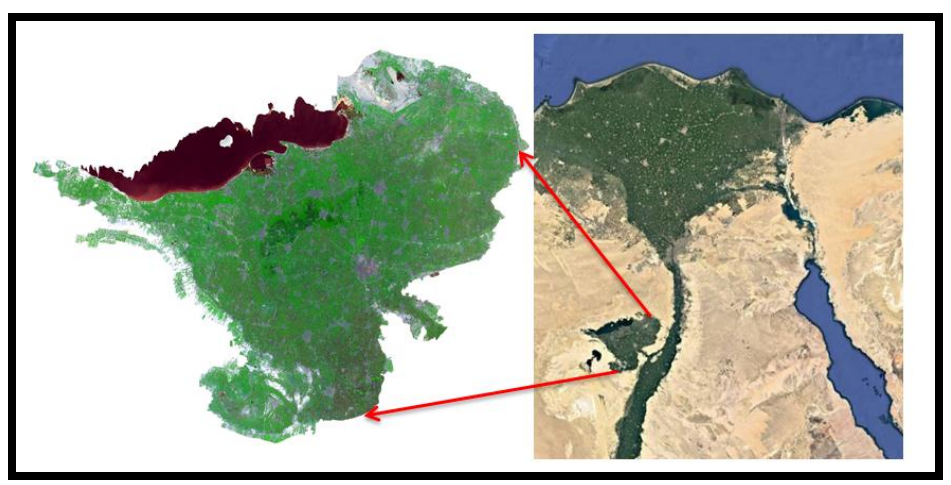

Fig (1): Study area. 


\section{Remote sensing and GIS work}

The digital elevation model (DEM) of the study area was extracted from the Shuttle Radar Topography Mission (SRTM) and a topographic map with a scale of 1:25,000 covering the study area using Arc-GIS 10.7.1 software (ESRI, 2003). The Landsat 8 (path 177 / row 40) image acquired in 2018 and SRTM data were processed in ENVI 5.5 software (ITT, 2017) to identify the geomorphology and landforms of the studied area (Fig, 2) according to the approach developed by Dobos et al. (2002). The map legend was designed according to Zinck and Valenzuela (1990). ArcMap 10.7.1 software was used to display and produce geomorphic map of the study area (Fig, 2) with help of its DEM features and field observations (ESRI, 2014).

\section{Field work}

Reconnaissance soil survey was conducted throughout the investigated area in order to acquire an appreciation of its broad soil patterns and characteristic landscape. The primary mapping units resulting from analysis of the DEM and interpretation information gained during unsupervised classification of Landsat images were verified.

Thirty soil profiles were chosen from two sample areas representing the geomorphic and landform units of the studied area (Fig, 2). The soil profile's locations and elevations were defined in the field by using GPS system. The morphological description of these soil profiles was recorded on the basis outlined by FAO (2006). Soil samples were collected based on the vertical variations of each soil profile for the laboratory analyses of soil physical and chemical properties.

\section{Laboratory analyses}

Particle size distribution, electrical conductivity (EC), pH, organic matter $(\mathrm{OM})$, calcium carbonate $\left(\mathrm{CaCO}_{3}\right)$, gypsum contents, cation exchange capacity (CEC), exchangeable $\mathrm{Na}^{+}$ percentage (ESP) were determined according to Burt and Soil Survey Staff (2014). Identify class terms of each soil property was done according to Soil Science Division Staff (2017).

\section{Soil classification}

The soils of the studied area were classified up to sub great group level based on Soil Survey Staff (2014).

\section{RESULTUS AND DISCUSSION Geomorphology}

Produced DEM map of the study area presented in Fig (3) indicated that, the elevation for the whole of the study area is varied between $-53 \mathrm{~m}$ below sea level (b.s.l.) and increased gradually to $65 \mathrm{~m}$ above sea level (a.s.I.).

The interpretation of satellite images with the aid of produced Digital Elevation Model (DEM), topographic maps and field observations indicated that, the study area has five main geomorphic units with different landforms (Fig, 2). These geomorphic units are: 1) Recent river terraces (High, Moderate and Low), 2) Basins (Overflow basins and Decantation basins), 3) Old river terraces (High, moderately high, Moderate and Low), 4) Former lake bed, and 5) Water bodies (Fish ponds and Lake Qaroun). These units with their representative soil profiles and their areas are presented in Table (1). 
M.S. Amira, er al.,

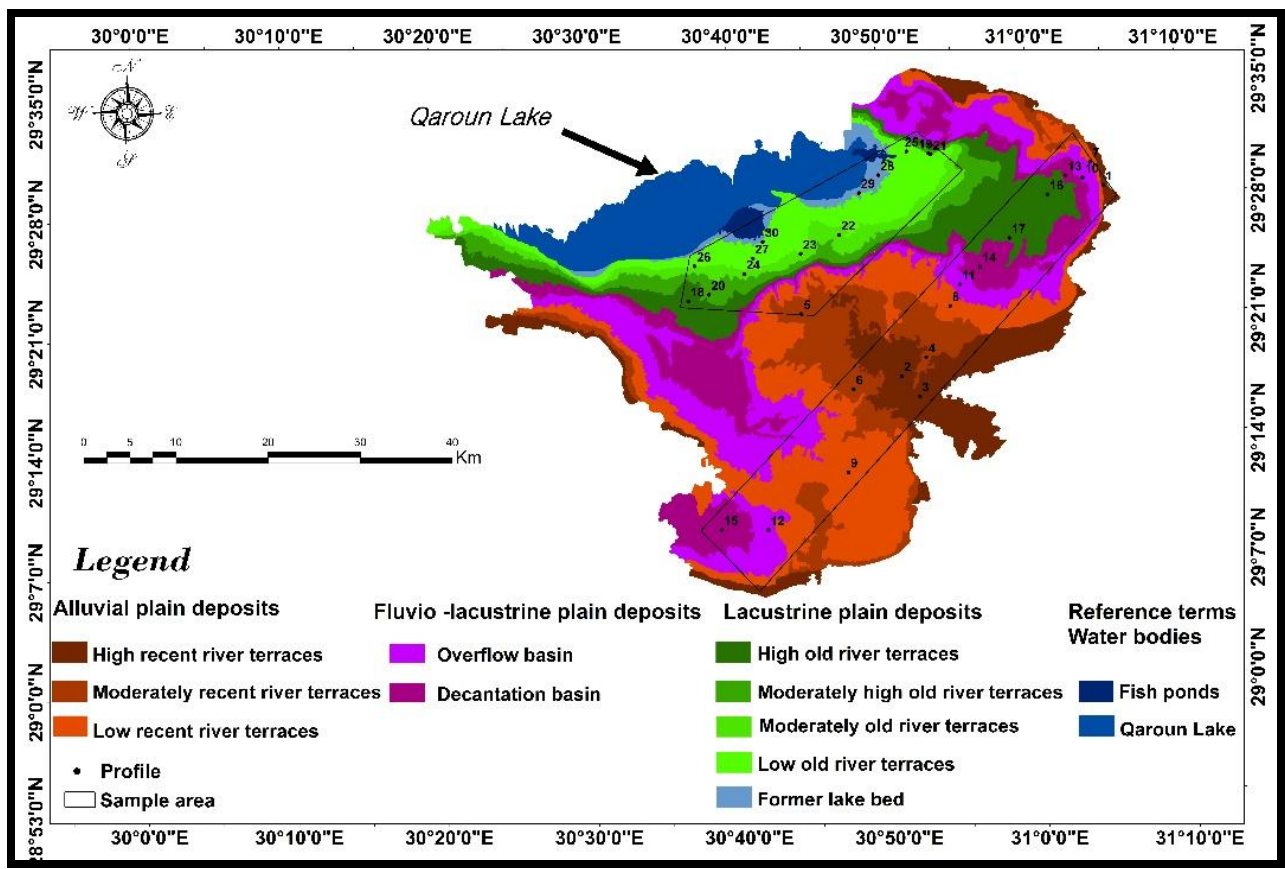

Fig (2): Geomorphic map and representative soil profiles of the study area.

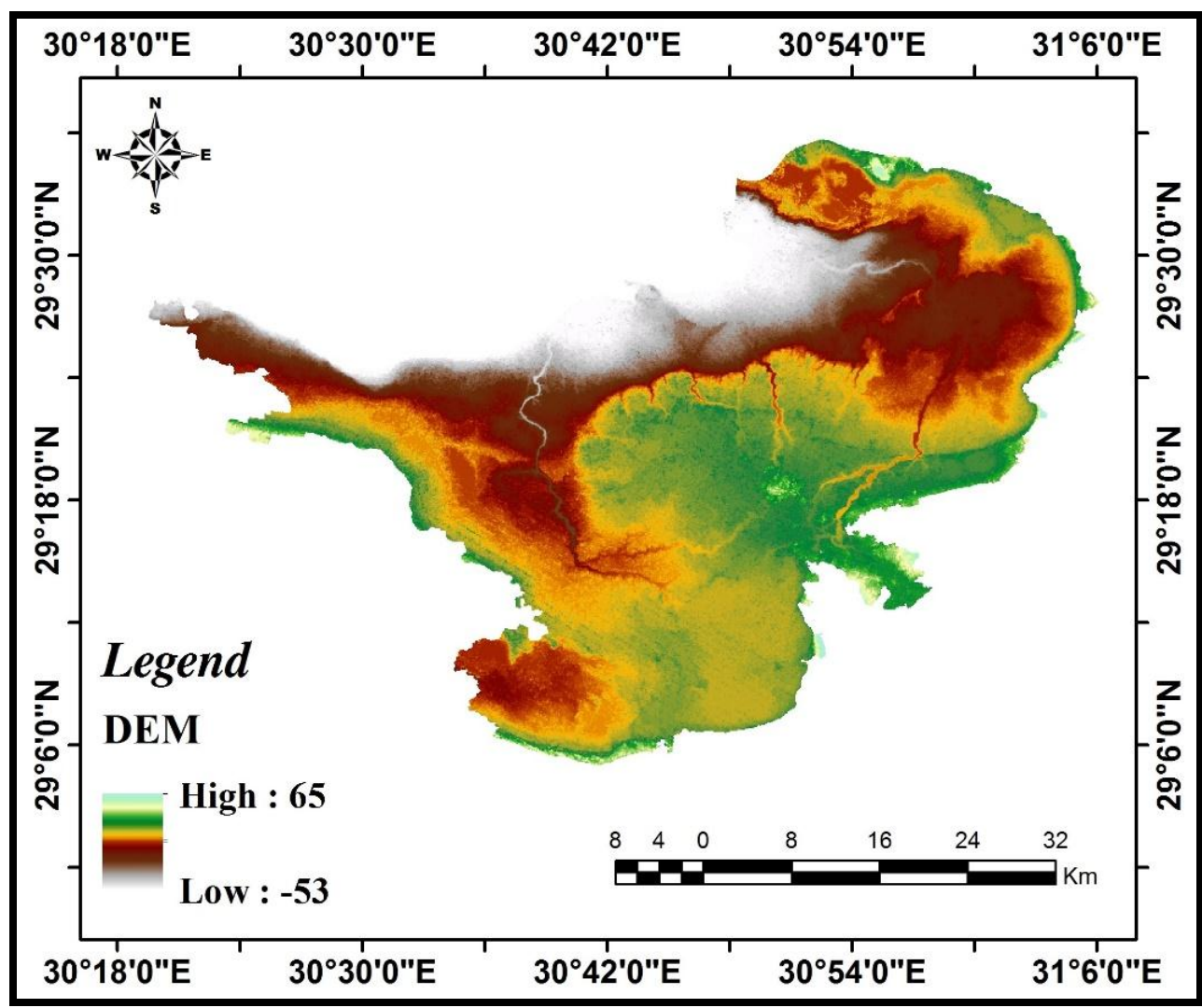

Fig (3): Digital Elevation Model (DEM) of the study area. 
Table (1): Detailed geomorphic units of the study area.

\begin{tabular}{|c|c|c|c|c|c|c|}
\hline \multirow{2}{*}{ Geomorphic units } & \multirow{2}{*}{ Landform } & \multirow{2}{*}{$\begin{array}{c}\text { Profiles } \\
\text { No. }\end{array}$} & \multicolumn{4}{|c|}{ Area } \\
\hline & & & $\mathrm{Km}^{2}$ & Fed & $\%$ & Total \\
\hline \multirow{3}{*}{$\begin{array}{l}\text { Recent river } \\
\text { terraces }\end{array}$} & High & $1,2,3$ & 215.77 & 51373.94 & 10.09 & \multirow{3}{*}{39.52} \\
\hline & Moderate & $4,5,6$ & 205.83 & 49007.17 & 9.62 & \\
\hline & Low & $7,8,9$ & 423.45 & 100820.69 & 19.8 & \\
\hline \multirow{2}{*}{ Basins } & Overflow & $10,11,12$ & 301 & 71667.01 & 14.08 & \multirow{2}{*}{25.31} \\
\hline & Decantation & $13,14,15$ & 240.28 & 57209.16 & 11.24 & \\
\hline \multirow{4}{*}{ Old river terraces } & High & $16,17,18$ & 187.33 & 44601.95 & 8.76 & \multirow{4}{*}{22.33} \\
\hline & Moderately high & $19,20,21$ & 87.48 & 20828.53 & 4.09 & \\
\hline & Moderate & $22,23,24$ & 67.56 & 16085.93 & 3.16 & \\
\hline & Low & $25,26,27$ & 135.27 & 32207.48 & 6.33 & \\
\hline Former lake bed & Former lake bed & $28,29,30$ & 32.21 & 7669.32 & 1.51 & 1.51 \\
\hline \multirow{2}{*}{ Water bodies } & Fish ponds & & 16.2 & 3857.61 & 0.76 & \multirow{2}{*}{11.33} \\
\hline & Qaroun Lake & & 226.15 & 53844.38 & 10.57 & \\
\hline \multicolumn{3}{|c|}{ Total } & 2138.53 & 509173.81 & 100 & 100 \\
\hline
\end{tabular}

\section{Soil morphology}

The morphological features of the studied soil profiles presented in Table (2) revealed that, the elevation of the studied soils is between - $44 \mathrm{~m}$ below sea level to $29 \mathrm{~m}$ above sea level. Most of the studied soils have almost flat topography with very deep soil materials. Some soils that having moderately high-water table levels, are moderately deep and imperfectly drained. The main hue notation of the studied soil color is around brown notation (10YR). The soils have mostly moderate medium subangular to granular

structure with hard dry and firm moist consistency. The studied soils are moderately calcareous.

\section{Physiochemical properties}

The physiochemical properties of the studied soil profiles and their weighted profile means (wpm) are registered in Table (3). Data in Table (3) show that, most of the studied soils have clay loam texture grade and some have loam to sandy loam ones. Most of these soils are non- to slightly-saline having a slightly alkaline reaction and slightly sodic effect. Some soils of old river terraces and all soils of Overflow basin, as well as Former lake bed, are moderately saline having sodicity effect. On the other hand, most of the studied soils are moderately calcareous having $<10 \% \mathrm{CaCO}_{3}$ content. Only, the soils of Overflow basin are highly calcareous having $>10 \% \mathrm{CaCO}_{3}$ content. All studied soils have low OM and gypsum contents. 
M.S. Amira, er al.,

Table (2): Morphological features of the studied soil profiles.

\begin{tabular}{|c|c|c|c|c|c|c|c|c|c|c|}
\hline \multirow{2}{*}{\multicolumn{2}{|c|}{$\begin{array}{l}\text { Geomorphic and } \\
\text { landform units }\end{array}$}} & \multirow{5}{*}{\begin{tabular}{|c|}
$\begin{array}{c}\text { Profile } \\
\text { No. }\end{array}$ \\
1 \\
\end{tabular}} & \multirow{5}{*}{$\begin{array}{c}\begin{array}{c}\text { Elevation } \\
\mathrm{m}\end{array} \\
26\end{array}$} & \multirow{3}{*}{\begin{tabular}{|c|}
$\begin{array}{c}\text { Depth } \\
\text { cm }\end{array}$ \\
$0-30$ \\
\end{tabular}} & \multicolumn{2}{|c|}{ Color } & \multirow{3}{*}{$\begin{array}{c}\text { Structure }^{1} \\
2 \mathrm{~m} \mathrm{sbk}\end{array}$} & \multicolumn{2}{|c|}{ Consistence $^{2}$} & \multirow{3}{*}{$\begin{array}{c}\text { Boundary }^{3} \\
\text { gradual s }\end{array}$} \\
\hline & & & & & \multirow{2}{*}{\begin{tabular}{|c|} 
Dry \\
10YR 6/3 \\
\end{tabular}} & \multirow{2}{*}{\begin{tabular}{|c|} 
Moist \\
10YR 6/2
\end{tabular}} & & Dry & Moist & \\
\hline \multirow{31}{*}{ 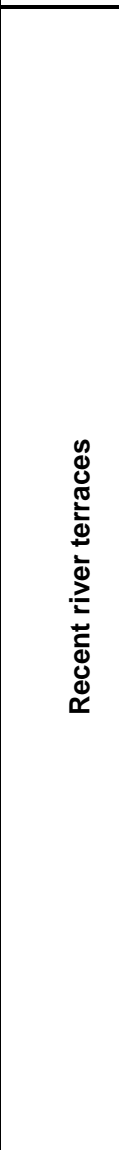 } & \multirow{9}{*}{ 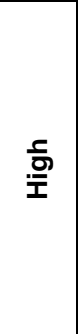 } & & & & & & & hard & firm & \\
\hline & & & & $30-70$ & 10YR 5/3 & 10YR 5/2 & 2 to $s \mathrm{~m}$ sbk & v hard & v firm & abrupt \\
\hline & & & & $70-150$ & 10YR 4/2 & 10YR 4/3 & $2 \mathrm{~m}$ an to sbk & hard & firm & - \\
\hline & & \multirow{3}{*}{2} & \multirow{3}{*}{29} & $0-25$ & 10YR4/3 & 10YR $3 / 3$ & $2 \mathrm{~m} \mathrm{sbk}$ & hard & firm & gradual s \\
\hline & & & & $25-90$ & \begin{tabular}{|l|} 
10YR 4/1 \\
\end{tabular} & 10YR3/1 & $2 \mathrm{~m} \mathrm{sbk}$ & v hard & v firm & gradual s \\
\hline & & & & $90-150$ & 10YR 4/2 & 10YR $4 / 3$ & $2 \mathrm{~m} \mathrm{sbk}$ & hard & firm & - \\
\hline & & \multirow{3}{*}{3} & \multirow{3}{*}{28} & $0-30$ & 10YR 4/4 & \begin{tabular}{|l}
$10 \mathrm{YR} 3 / 4$ \\
\end{tabular} & $2 \mathrm{~m} \mathrm{sbk}$ & hard & firm & gradual s \\
\hline & & & & $30-60$ & 10YR3/2 & 10YR 2/2 & $2 \mathrm{~m} \mathrm{sbk}$ & v hard & v firm & abrupt \\
\hline & & & & $60-150$ & \begin{tabular}{|l|} 
10YR 4/2 \\
\end{tabular} & 10YR 3/2 & $2 \mathrm{~m} \mathrm{sbk}$ & hard & firm & - \\
\hline & & & & $0-30$ & \begin{tabular}{|l|}
$10 Y R ~ 6 / 4$ \\
\end{tabular} & $\begin{array}{l}\text { 10YR 5/4 } \\
\end{array}$ & $2 \mathrm{~m} \mathrm{sbk}$ & hard & firm & clear \\
\hline & & 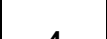 & & $30-55$ & \begin{tabular}{|l|}
$10 Y R ~ 4 / 3$ \\
\end{tabular} & 10YR 4/2 & $2 \mathrm{~m} \mathrm{sbk}$ & hard & firm & gradual s \\
\hline & & 4 & 19 & $55-110$ & 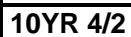 & \begin{tabular}{|l|} 
10YR 4/1 \\
\end{tabular} & $2 \mathrm{~m} \mathrm{sbk}$ & hard & firm & clear \\
\hline & $\frac{2}{0}$ & & & $110-150$ & \begin{tabular}{|l|} 
10YR 5/4 \\
\end{tabular} & \begin{tabular}{|l|}
$10 Y R ~ 5 / 3$ \\
\end{tabular} & $2 \mathrm{~m} \mathrm{sbk}$ & hard & v firm & \\
\hline & సँ & 5 & 10 & $0-35$ & 10YR $6 / 3$ & 10YR $6 / 2$ & $2 \mathrm{~m} \mathrm{sbk}$ & hard & firm & abrupt \\
\hline & $\frac{\bar{\sigma}}{0}$ & 5 & 18 & $35-100$ & 10YR 6/4 & 10YR $6 / 3$ & $2 \mathrm{~m} \mathrm{sbk}$ & soft & friable & - \\
\hline & ㅁ & & & $0-25$ & 10YR 4/1 & 10YR3/1 & $2 \mathrm{~m} \mathrm{sbk}$ & hard & firm & abrupt \\
\hline & & & & $25-50$ & 10YR 4/3 & 10YR 4/2 & $2 \mathrm{~m} \mathrm{sbk}$ & hard & firm & abrupt \\
\hline & & 6 & 18 & $50-100$ & 10YR 6/6 & 10YR5/6 & $2 \mathrm{~m} \mathrm{sbk}$ & hard & firm & gradual s \\
\hline & & & & $100-150$ & 10YR 3/1 & 10YR2/1 & $2 \mathrm{~m} \mathrm{sbk}$ & hard & firm & - \\
\hline & & & & $0-30$ & 10YR 5/2 & 10YR 5/1 & $2 \mathrm{~m} \mathrm{sbk}$ & hard & firm & gradual s \\
\hline & & 7 & 15 & $30-60$ & 10YR 3/2 & 10YR 3/1 & $2 \mathrm{~m} \mathrm{sbk}$ & hard & firm & gradual s \\
\hline & & $f$ & 15 & $60-100$ & 10YR 4/1 & 10YR 3/1 & $2 \mathrm{~m} \mathrm{sbk}$ & hard & v firm & clear \\
\hline & & & & $100-150$ & 10YR 4/3 & 10YR 4/2 & $2 \mathrm{~m} \mathrm{sbk}$ & hard & v firm & - \\
\hline & & & & $0-25$ & 10YR 4/2 & 10YR3/2 & $2 \mathrm{~m} \mathrm{sbk}$ & v hard & v firm & gradual s \\
\hline & & & & $25-50$ & 10YR 4/4 & 10YR3/4 & $2 \mathrm{~m} \mathrm{sbk}$ & hard & firm & clear \\
\hline & 9 & 8 & 12 & $50-120$ & 10YR 7/6 & 10YR6/6 & $2 \mathrm{~m} \mathrm{sbk}$ & hard & firm & gradual s \\
\hline & & & & $120-150$ & 10YR4/6 & 10YR3/6 & $\mathrm{sm}$ an & hard & firm & - \\
\hline & & & & $0-30$ & 10YR 7/6 & 10YR 6/6 & $2 \mathrm{~m} \mathrm{sbk}$ & hard & firm & gradual s \\
\hline & & & & $30-50$ & \begin{tabular}{|l} 
10YR $6 / 6$ \\
\end{tabular} & 10YR 6/4 & $2 \mathrm{~m} \mathrm{sbk}$ & hard & firm & clear \\
\hline & & 9 & 15 & $50-110$ & \begin{tabular}{|l|} 
10YR $6 / 4$ \\
\end{tabular} & \begin{tabular}{|l|} 
10YR $6 / 3$ \\
\end{tabular} & $2 \mathrm{~m} \mathrm{sbk}$ & hard & firm & gradual s \\
\hline & & & & $110-150$ & 10YR 5/4 & 10YR 5/3 & $1 \mathrm{f} \mathrm{gr}$ & soft & v friable & - \\
\hline & & & & $0-15$ & 10YR 7/4 & 10YR $7 / 3$ & $2 \mathrm{~m} \mathrm{sbk}$ & v hard & v firm & clear \\
\hline & & & & $15-40$ & 10YR 7/6 & 10YR 7/4 & $1 \mathrm{fgr}$ & soft & $v$ friable & clear \\
\hline & & 10 & 2 & $40-70$ & \begin{tabular}{|l|}
$10 Y R 6 / 8$ \\
\end{tabular} & 10YR 6/6 & $1 \mathrm{fgr}$ & s hard & firm & clear \\
\hline & & & & $70-110$ & 10YR $7 / 8$ & 10YR 6/8 & $1 \mathrm{fgr}$ & s hard & firm & - \\
\hline & 은 & & & $0-20$ & 10YR 6/6 & $\begin{array}{l}\text { 10YR } 6 / 5 \\
\end{array}$ & $1 \mathrm{f} \mathrm{sbk}$ & s hard & firm & clear \\
\hline & \$) & 11 & 7 & $20-50$ & 10YR 7/4 & 10YR 6/4 & $1 \mathrm{~m} \mathrm{sbk}$ & hard & friable & clear \\
\hline & & & & $50-120$ & 10YR 8/6 & $\begin{array}{l}\text { 10YR } 8 / 4 \\
\end{array}$ & $2 \mathrm{~m} \mathrm{sbk}$ & hard & firm & - \\
\hline & & & & $0-25$ & 10YR 6/8 & 10YR 5/8 & $2 \mathrm{~ms}$ & soft & friable & clear \\
\hline & & 12 & 6 & $25-50$ & 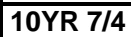 & 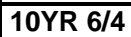 & $1 \mathrm{~m} \mathrm{sbk}$ & hard & friable & clear \\
\hline & & & & $50-140$ & 10YR 7/6 & 10YR 6/6 & $2 \mathrm{~ms}$ & soft & friable & - \\
\hline & & & & $0-25$ & 10YR $5 / 3$ & 10YR 5/2 & $2 \mathrm{~m} \mathrm{sbk}$ & hard & firm & gradual s \\
\hline & & & & $25-65$ & 10YR 5/4 & 10YR 5/3 & 2 to $\mathrm{s} \mathrm{m} \mathrm{sbk}$ & v hard & v firm & gradual s \\
\hline & & 13 & 2 & $65-110$ & 10YR 5/6 & \begin{tabular}{|l} 
10YR 5/7 \\
\end{tabular} & $1 \mathrm{fgr}$ & soft & friable & clear \\
\hline & & & & $110-150$ & 10YR 3/2 & 10YR 3/1 & $2 \mathrm{~m} \mathrm{sbk}$ & hard & firm & - \\
\hline & & & & $0-30$ & 10YR $6 / 2$ & 10YR 6/1 & $2 \mathrm{~ms}$ & soft & friable & clear \\
\hline & 垔 & & & $30-60$ & 10YR 4/2 & 10YR 4/1 & $2 \mathrm{~m} \mathrm{sbk}$ & hard & firm & clear \\
\hline & 宽 & 14 & $(-1)$ & $60-110$ & \begin{tabular}{|l|} 
10YR 5/3 \\
\end{tabular} & $\begin{array}{l}\text { 10YR 5/2 } \\
\end{array}$ & $2 \mathrm{~m} \mathrm{sbk}$ & hard & v firm & gradual s \\
\hline & 0 & & & $110-150$ & \begin{tabular}{|l|} 
10YR 5/4 \\
\end{tabular} & 10YR 5/3 & $2 \mathrm{~m} \mathrm{sbk}$ & hard & v firm & - \\
\hline & & & & $0-20$ & 10YR $7 / 6$ & 10YR6/6 & $2 \mathrm{~ms}$ & soft & friable & clear \\
\hline & & 1 & 1 & $20-50$ & \begin{tabular}{|l|l} 
10YR 4/4 \\
\end{tabular} & 10YR 3/4 & $2 \mathrm{~m} \mathrm{sbk}$ & hard & firm & clear \\
\hline & & 5 & 1 & $50-120$ & 10YR 4/1 & 10YR 3/1 & $2 \mathrm{~m} \mathrm{sbk}$ & hard & firm & diffuse \\
\hline & & & & $120-150$ & \begin{tabular}{|l|} 
10YR 4/1 \\
\end{tabular} & \begin{tabular}{|l} 
10YR 3/1 \\
\end{tabular} & $2 \mathrm{~m} \mathrm{sbk}$ & hard & v firm & - \\
\hline
\end{tabular}

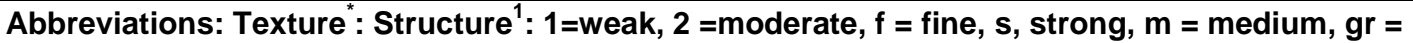
granular, an = angular ,sbk = subangular blocky, $\mathbf{s g}=$ single grains; Consistence $^{2}$ : $\mathrm{v}=$ very, Boundary ${ }^{3}: \mathrm{s}=$ smooth : $\mathrm{ms}=$ massive 
Table (2): Cont.

\begin{tabular}{|c|c|c|c|c|c|c|c|c|c|c|}
\hline \multirow{2}{*}{\multicolumn{2}{|c|}{$\begin{array}{l}\text { Geomorphic and } \\
\text { landform units }\end{array}$}} & \multirow{2}{*}{$\begin{array}{l}\text { Profile } \\
\text { No. }\end{array}$} & \multirow{2}{*}{$\begin{array}{c}\text { Elevation } \\
\mathbf{m}\end{array}$} & \multirow{2}{*}{$\begin{array}{l}\text { Depth } \\
\text { cm }\end{array}$} & \multicolumn{2}{|c|}{ Color } & \multirow{2}{*}{ Structure $^{1}$} & \multicolumn{2}{|c|}{ Consistence $^{2}$} & \multirow{2}{*}{$\underset{3}{B}$} \\
\hline & & & & & Dry & Moist & & Dry & Moist & \\
\hline \multirow{42}{*}{ 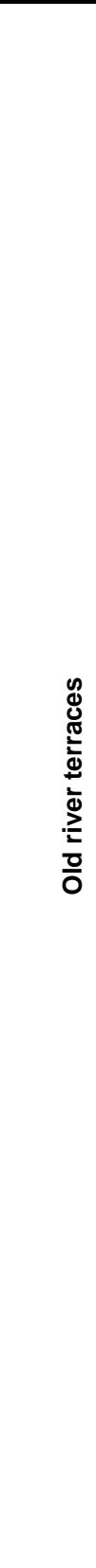 } & \multirow{10}{*}{ 宁 } & \multirow{4}{*}{16} & \multirow{4}{*}{$(-10)$} & $0-20$ & 10YR 5/3 & 10YR 5/4 & $2 \mathrm{~m} \mathrm{sbk}$ & hard & firm & gradual s \\
\hline & & & & $20-60$ & \begin{tabular}{|l|}
$10 Y R ~ 4 / 3$ \\
\end{tabular} & 10YR 4/2 & $2 \mathrm{~m} \mathrm{sbk}$ & hard & firm & gradual s \\
\hline & & & & $60-115$ & 10YR 3/2 & 10YR 3/1 & $2 \mathrm{~m} \mathrm{sbk}$ & hard & v firm & clear \\
\hline & & & & $115-150$ & 10YR 4/4 & 10YR 4/3 & $2 \mathrm{~m}$ sbk & hard & v firm & - \\
\hline & & \multirow{3}{*}{17} & \multirow{3}{*}{$(-4)$} & $0-15$ & 10YR 6/4 & 10YR 6/3 & $2 \mathrm{~m} \mathrm{sbk}$ & hard & firm & clear \\
\hline & & & & $15-80$ & 10YR 5/3 & 10YR 5/2 & 2 to $\mathrm{s} \mathrm{m} \mathrm{sbk}$ & v hard & v firm & diffuse \\
\hline & & & & $80-150$ & 10YR 5/3 & 10YR 5/2 & $2 \mathrm{~m}$ sbk & v hard & v firm & - \\
\hline & & \multirow{3}{*}{18} & \multirow{3}{*}{$(-5)$} & $0-20$ & \begin{tabular}{|l|} 
10YR 4/2 \\
\end{tabular} & 10YR 3/2 & $2 \mathrm{~m} \mathrm{sbk}$ & v hard & v firm & clear \\
\hline & & & & $20-90$ & 10YR 3/1 & 10YR 2/1 & $2 \mathrm{~m} \mathrm{sbk}$ & hard & firm & clear \\
\hline & & & & $90-150$ & 10YR 6/6 & 10YR 5/6 & $2 \mathrm{~m}$ sbk & hard & firm & \\
\hline & & & & $0-20$ & 10YR 7/6 & 10YR 6/6 & $2 \mathrm{~m} \mathrm{sbk}$ & hard & firm & clear \\
\hline & & 19 & $(-16)$ & $20-55$ & 10YR 6/6 & 10YR 5/6 & $1 \mathrm{~m} \mathrm{sbk}$ & hard & firm & clear \\
\hline & & & & $55-100$ & 10YR 8/6 & 10YR $7 / 6$ & $2 \mathrm{~m} \mathrm{sbk}$ & hard & v firm & \\
\hline & 동 & & & $0-25$ & \begin{tabular}{|l|}
$10 Y R ~ 5 / 3$ \\
\end{tabular} & 10YR 5/2 & $2 \mathrm{~m} \mathrm{sbk}$ & hard & firm & clear \\
\hline & & ?0 & (20) & $25-60$ & 10YR 4/2 & 10YR 4/1 & $1 \mathrm{~m} \mathrm{sbk}$ & hard & firm & gradual s \\
\hline & & 20 & $(-28)$ & $60-100$ & 10YR 4/3 & 10YR 4/2 & $2 \mathrm{~m} \mathrm{sbk}$ & hard & v firm & clear \\
\hline & $\frac{\pi}{6}$ & & & \begin{tabular}{|l|}
$100-150$ \\
\end{tabular} & 10YR 3/2 & 10YR 3/1 & $2 \mathrm{~m} \mathrm{sbk}$ & hard & v firm & - \\
\hline & 8 & & & $0-20$ & 10YR 5/2 & 10YR 4/2 & $2 \mathrm{~m} \mathrm{sbk}$ & hard & firm & clear \\
\hline & & 21 & $(-14)$ & $20-50$ & 10YR 3/2 & 10YR 3/1 & $1 \mathrm{~m} \mathrm{sbk}$ & hard & firm & gradual s \\
\hline & & 21 & $(-14)$ & \begin{tabular}{|l|}
$50-110$ \\
\end{tabular} & \begin{tabular}{|l|} 
10YR 4/2 \\
\end{tabular} & 10YR 4/1 & $2 \mathrm{~m} \mathrm{sbk}$ & hard & firm & clear \\
\hline & & & & $110-150$ & 10YR 5/3 & 10YR 5/2 & $2 \mathrm{~m}$ sbk & hard & v firm & - \\
\hline & & & & $0-25$ & 10YR 4/2 & 10YR 4/1 & $2 \mathrm{~m} \mathrm{sbk}$ & hard & firm & gradual s \\
\hline & & 22 & $(-25)$ & $25-70$ & \begin{tabular}{|l|} 
10YR 3/3 \\
\end{tabular} & 10YR 3/2 & $2 \mathrm{~m} \mathrm{sbk}$ & hard & firm & clear \\
\hline & & & & \begin{tabular}{|l|}
$70-130$ \\
\end{tabular} & \begin{tabular}{|l|} 
10YR 3/1 \\
\end{tabular} & 10YR 2/1 & $2 \mathrm{~m} \mathrm{sbk}$ & v hard & v firm & - \\
\hline & & & & $0-30$ & 10YR 6/4 & 10YR $6 / 3$ & $2 \mathrm{~m}$ sbk & hard & firm & gradual s \\
\hline & $\Phi$ & 23 & $(-26)$ & $30-50$ & 10YR 5/4 & 10YR $5 / 3$ & $2 \mathrm{~m} \mathrm{sbk}$ & hard & firm & gradual s \\
\hline & $\frac{\pi}{2}$ & 23 & $(-26)$ & $50-100$ & 10YR 5/3 & 10YR 5/2 & $2 \mathrm{~m} \mathrm{sbk}$ & hard & v firm & gradual s \\
\hline & 음 & & & $100-150$ & 10YR 5/6 & 10YR 5/4 & $2 \mathrm{~m} \mathrm{sbk}$ & hard & v firm & - \\
\hline & & & & $0-30$ & 10YR 4/3 & 10YR 3/3 & $2 \mathrm{~m} \mathrm{sbk}$ & hard & firm & clear \\
\hline & & & & $30-60$ & 10YR 5/4 & 10YR 4/4 & $2 \mathrm{~m} \mathrm{sbk}$ & hard & firm & gradual s \\
\hline & & 24 & $(-25)$ & \begin{tabular}{|l|}
$60-120$ \\
\end{tabular} & \begin{tabular}{|l|} 
10YR $6 / 6$ \\
\end{tabular} & 10YR 5/6 & $2 \mathrm{~m} \mathrm{sbk}$ & hard & firm & gradual s \\
\hline & & & & \begin{tabular}{|l|}
$120-150$ \\
\end{tabular} & 10YR 5/6 & 10YR 5/4 & $2 \mathrm{~m} \mathrm{sbk}$ & hard & v firm & - \\
\hline & & & & $0-10$ & 10YR 6/2 & 10YR 5/2 & $2 \mathrm{~m} \mathrm{sbk}$ & v hard & v firm & gradual s \\
\hline & & 25 & $(-10)$ & $10--40$ & 10YR 4/2 & 10YR 3/2 & 2 to $\mathrm{s} \mathrm{m} \mathrm{sbk}$ & v hard & v firm & gradual s \\
\hline & & 25 & $(-40)$ & $40-110$ & 10YR 4/1 & 10YR 4/2 & $2 \mathrm{~m} \mathrm{sbk}$ & v hard & v firm & clear \\
\hline & & & & \begin{tabular}{|l|}
$110-150$ \\
\end{tabular} & \begin{tabular}{|l|}
$10 Y R ~ 4 / 3$ \\
\end{tabular} & 10YR 3/3 & $2 \mathrm{~m}$ an to sbk & hard & firm & - \\
\hline & 3 & 26 & $(25)$ & $0-25$ & 10YR 5/4 & 10YR 5/3 & $2 \mathrm{~m} \mathrm{sbk}$ & v hard & v firm & clear \\
\hline & ( & 20 & (-30) & $25-70$ & 10YR 5/2 & 10YR 5/1 & $2 \mathrm{~m}$ an to sbk & hard & firm & - \\
\hline & & & & $0-20$ & 10YR 5/3 & 10YR 4/3 & $2 \mathrm{~m}$ sbk & v hard & v firm & clear \\
\hline & & 27 & $(-37)$ & \begin{tabular}{|l|}
$20--40$ \\
\end{tabular} & \begin{tabular}{|l|} 
10YR 5/4 \\
\end{tabular} & 10YR 4/4 & $2 \mathrm{~m}$ an to sbk & hard & firm & gradual s \\
\hline & & 27 & $(-37)$ & $40-115$ & \begin{tabular}{|l|} 
10YR 4/2 \\
\end{tabular} & 10YR 3/2 & $1 \mathrm{~m} \mathrm{sbk}$ & hard & firm & gradual s \\
\hline & & & & \begin{tabular}{|l|}
$115-150$ \\
\end{tabular} & \begin{tabular}{|l|} 
10YR 4/1 \\
\end{tabular} & 10YR 3/1 & $2 \mathrm{~m}$ an & hard & v firm & - \\
\hline & & & & $0-10$ & 10YR 7/8 & 10YR 7/6 & s m sbk & hard & firm & gradual s \\
\hline & & ח & & $10--40$ & \begin{tabular}{|l|} 
10YR 6/6 \\
\end{tabular} & 10YR 6/8 & $\mathrm{sg}$ & \begin{tabular}{|l|} 
loose \\
\end{tabular} & Toose & clear \\
\hline & & 28 & $(-41)$ & \begin{tabular}{|l|}
$40-100$ \\
\end{tabular} & \begin{tabular}{|l|} 
10YR 4/2 \\
\end{tabular} & 10YR 3/2 & $2 m$ an & hard & firm & clear \\
\hline & ర్ల & & & $100-150$ & 10YR 3/2 & 10YR 3/1 & $1 \mathrm{~m}$ an & hard & firm & - \\
\hline$\stackrel{0}{0}$ & $\stackrel{0}{0}$ & & & $0-15$ & 10YR 7/6 & 10YR 6/6 & $2 \mathrm{~m}$ sbk & hard & firm & gradual s \\
\hline 莹 & 흠 & 29 & & $15--50$ & 10YR 8/4 & 10YR $7 / 4$ & $\mathrm{sg}$ & s hard & friable & clear \\
\hline$=$ & $\overline{\bar{\sigma}}$ & 29 & $(-44)$ & $50-110$ & 10YR 4/1 & 10YR 3/1 & $2 \mathrm{~m}$ an to sbk & hard & firm & clear \\
\hline$=$ & $\underline{\underline{E}}$ & & & 110-150 & 10YR 5/4 & 10YR 4/4 & $2 \mathrm{~m}$ an to $\mathrm{sbk}$ & hard & firm & - \\
\hline 훈 & 훈 & & & $0-30$ & 10YR 4/6 & 10YR3/6 & s m sbk & hard & firm & clear \\
\hline & & 30 & (4) & $30--80$ & 10YR 8/6 & 10YR 7/6 & $2 \mathrm{~m}$ an & hard & firm & gradual s \\
\hline & & 30 & $(-42)$ & $80-100$ & 10YR 6/6 & 10YR 5/6 & $2 \mathrm{~m} \mathrm{sbk}$ & hard & firm & clear \\
\hline & & & & \begin{tabular}{|l|}
$100-150$ \\
\end{tabular} & 10YR 4/2 & 10YR 3/2 & 1 an to sbk & hard & firm & - \\
\hline
\end{tabular}


M.S. Amira, er al.,

Table (3): Some physical and chemical properties of studied soil profiles.

\begin{tabular}{|c|c|c|c|c|c|c|c|c|c|c|c|c|c|c|}
\hline \multirow{2}{*}{\multicolumn{2}{|c|}{ Landform }} & \multirow{2}{*}{$\begin{array}{l}P^{1} \\
\text { No }\end{array}$} & \multirow{2}{*}{$\begin{array}{c}\text { Depth } \\
\text { cm }\end{array}$} & \multicolumn{3}{|c|}{$\begin{array}{c}\text { Particle size } \\
\text { distribution \% }\end{array}$} & \multirow{2}{*}{$\begin{array}{l}\text { Texture } \\
\text { class }^{3}\end{array}$} & \multirow{2}{*}{$\begin{array}{c}\mathrm{CaCO}_{3} \\
\%\end{array}$} & \multirow[t]{2}{*}{$E C^{4}$} & \multirow[t]{2}{*}{$\mathrm{pH}^{5}$} & \multirow[t]{2}{*}{ CEC $^{6}$} & \multirow[t]{2}{*}{$\begin{array}{c}\text { Gyp }^{7} \\
\%\end{array}$} & \multirow{2}{*}{$\begin{array}{l}\text { O. M } \\
\%\end{array}$} & \multirow{2}{*}{$\begin{array}{c}\text { ESP } \\
\%\end{array}$} \\
\hline & & & & Sand & \begin{tabular}{|l|} 
Silt \\
\end{tabular} & Clay & & & & & & & & \\
\hline \multirow{40}{*}{ 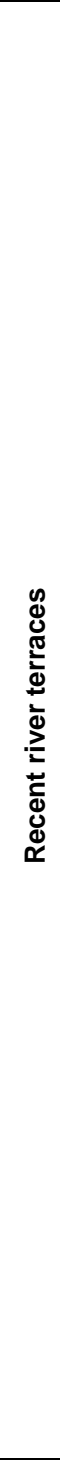 } & \multirow{12}{*}{ 듬 } & & $0-30$ & 34.6 & 30.3 & 35.1 & Clay loam & 7.2 & 2.9 & \begin{tabular}{|l|}
7.79 \\
\end{tabular} & 23.6 & 0.24 & 0.24 & \begin{tabular}{|l|}
10.7 \\
\end{tabular} \\
\hline & & 1 & $30-70$ & 19.9 & 37.5 & 42.6 & Clay & 6.3 & 2.4 & 7.84 & 27.5 & 0.08 & 0.2 & \begin{tabular}{|l|}
7.9 \\
\end{tabular} \\
\hline & & 1 & $\begin{array}{l}70-150 \\
\end{array}$ & 22.5 & 39.2 & 38.3 & Clay loam & 6.7 & 2.6 & \begin{tabular}{|l|}
7.70 \\
\end{tabular} & 25.3 & 0.14 & 0.23 & 8.9 \\
\hline & & & WPM $^{2}$ & 24.2 & 37.0 & 38.8 & Clay loam & 6.7 & 2.6 & 7.75 & 25.5 & 0.14 & 0.22 & 9.0 \\
\hline & & & $0-25$ & 32.6 & 31.4 & 36.0 & Clay loam & 6.9 & 3.1 & 7.85 & 24.1 & 0.26 & .24 & 10.0 \\
\hline & & & $25-90$ & 24.0 & 36.6 & 39.4 & Clay loam & 5.5 & 2.7 & 7.83 & 25.9 & 0.07 & 0.31 & \begin{tabular}{|l|}
9.2 \\
\end{tabular} \\
\hline & & 2 & $90-150$ & 23.6 & 39.1 & 37.3 & Clay loam & 6.0 & 2.8 & 7.70 & 24.7 & 0.12 & 0.24 & 9.1 \\
\hline & & & WPM & 25.3 & \begin{tabular}{|l|}
36.7 \\
\end{tabular} & 38.0 & Clay loam & 5.9 & 2.8 & 7.78 & 25.1 & 0.21 & 0.27 & \begin{tabular}{|l|}
9.3 \\
\end{tabular} \\
\hline & & & $0-30$ & 35.0 & 29.0 & 36.0 & Clay loam & 8.8 & 2.8 & 7.88 & 24.1 & 0.25 & 0.26 & 11.0 \\
\hline & & 3 & $30-60$ & 21.2 & \begin{tabular}{|l|}
36.7 \\
\end{tabular} & 42.1 & Clay & 5.8 & 2.6 & 7.83 & 27.3 & 0.06 & 0.33 & \begin{tabular}{|l|}
8.3 \\
\end{tabular} \\
\hline & & 3 & $60-150$ & 22.7 & 38.2 & 39.1 & Clay loam & 5.2 & 2.7 & 7.77 & 25.7 & 0.15 & 0.25 & 7.5 \\
\hline & & & WPM & 24.9 & 36.1 & 39.0 & Clay loam & 6.0 & 2.7 & 7.80 & 25.7 & 0.2 & 0.3 & \begin{tabular}{|l|}
8.4 \\
\end{tabular} \\
\hline & & & $0-30$ & 32.4 & 34.5 & 33.1 & Clay loam & 4.2 & 2.5 & 7.81 & 22.5 & 0.17 & 0.28 & 10.8 \\
\hline & & & $30-55$ & 25.0 & 38.6 & 36.4 & Clay loam & 7.5 & 2.9 & 7.86 & 24.3 & 0.15 & 0.26 & \begin{tabular}{|l|}
10.1 \\
\end{tabular} \\
\hline & & 4 & $55-110$ & 27.7 & 37.8 & 34.5 & Clay loam & 5.7 & 3.0 & \begin{tabular}{|l|}
7.82 \\
\end{tabular} & 23.3 & 0.17 & 0.29 & \begin{tabular}{|l|}
9.1 \\
\end{tabular} \\
\hline & & & $110-150$ & 30.3 & 35.3 & 34.4 & Clay loam & 8.9 & 2.7 & \begin{tabular}{|l|}
7.76 \\
\end{tabular} & 23.2 & 0.14 & .25 & 9.9 \\
\hline & & & WPM & 28.9 & 36.6 & 34.5 & $\mathrm{Cla}$ & 6.6 & 2.8 & 7.81 & 23.3 & .16 & 27 & 8.9 \\
\hline & & & $0-35$ & 40.5 & 31.0 & 28.5 & $\mathrm{am}$ & 8.6 & 7.3 & 7.70 & 20.1 & .21 & .27 & 21.0 \\
\hline & $\frac{\pi}{2}$ & 5 & $35-100$ & 69.2 & 21.3 & 9.5 & Sandy loam & 13 & 2.9 & 7.85 & 10.0 & 0.24 & .14 & 10.1 \\
\hline & 흥 & & WPM & 59.2 & 24.7 & 16.1 & Sandy loam & 11.5 & 4.4 & 7.80 & 13.5 & 0.23 & .18 & 13.9 \\
\hline & & & $0-25$ & 34.7 & 31.3 & 34.0 & Clay loam & 7.6 & 2.2 & 7.81 & 23.0 & 0.19 & 0.26 & 9.6 \\
\hline & & & $25-50$ & 24.7 & \begin{tabular}{|l|}
37.2 \\
\end{tabular} & 38.1 & Clay loam & 8.3 & 2.9 & 7.85 & 25.2 & 0.2 & 0.28 & 10.0 \\
\hline & & 6 & $50-100$ & 24.9 & 38.5 & 36.6 & Clay loam & 5.8 & 2.8 & 7.84 & 24.4 & 0.23 & 0.26 & \begin{tabular}{|l|}
8.3 \\
\end{tabular} \\
\hline & & & $10-150$ & 25.8 & \begin{tabular}{|l|}
37.7 \\
\end{tabular} & 36.5 & Clay loam & 13.0 & 3.1 & 7.87 & 24.3 & 0.22 & 0.13 & \begin{tabular}{|l|}
9.5 \\
\end{tabular} \\
\hline & & & WPM & 26.8 & 36.8 & 36.4 & Clay & 8.9 & 2.8 & 7.84 & 24.3 & 0.22 & 0.22 & 9.2 \\
\hline & & & $0-30$ & 24.0 & 39.0 & 37.0 & Clay & 5.6 & 2.6 & 7.83 & 24.6 & 0.13 & 0.23 & \begin{tabular}{|l|}
9.9 \\
\end{tabular} \\
\hline & & & $30-60$ & 27.1 & 37.6 & 35.3 & am & 4.0 & 3.6 & 7.79 & 23.7 & 0.11 & 0.25 & 11.9 \\
\hline & & 7 & $60-100$ & 23.9 & 38.5 & 37.6 & lay loam & $\overline{3.8}$ & 3.0 & 7.78 & 24.9 & 0.13 & 0.27 & \begin{tabular}{|l|}
9.4 \\
\end{tabular} \\
\hline & & & $100-150$ & 32.1 & 35.3 & 32.6 & $\mathrm{am}$ & 5.0 & 2.8 & 7.75 & 22.2 & 0.15 & 0.24 & 11.4 \\
\hline & & & WPM & 27.3 & 37.3 & 35.4 & & 4.6 & 3.0 & 7.78 & 23.7 & 0.13 & .25 & 10.7 \\
\hline & & & -25 & 54.4 & 24.1 & 21.5 & S clay loam & 12.5 & 2.7 & 7.78 & 16.4 & 0.23 & 0.14 & 11.0 \\
\hline & & & $25-50$ & 46.7 & 26.8 & 26.5 & S clay loam & 11.2 & 2.9 & 7.85 & 19.0 & 0.24 & 0.16 & 10.0 \\
\hline & 0 & 8 & $50-120$ & 47.4 & 22.5 & 30.1 & S clay loam & 9.7 & 3.6 & 7.78 & 20.9 & 0.2 & 0.14 & 12.4 \\
\hline & & & $120-150$ & 51.1 & 21.6 & 27.3 & S clay loam & 13.8 & 4.0 & 7.70 & 19.4 & 0.22 & 0.15 & 11.4 \\
\hline & & & WPM & 49.2 & 23.3 & 27.5 & $\mathrm{am}$ & 11.2 & 3.4 & 7.77 & 19.5 & 0.22 & .15 & 11.6 \\
\hline & & & -30 & 54.2 & 23.3 & 22.5 & S clay & 13.3 & 2.9 & 7.85 & 16.9 & 0.24 & .16 & 9.2 \\
\hline & & & $30-50$ & 46.7 & 28.8 & 24.5 & & 14.5 & 3.0 & 7.82 & 18.0 & 0.26 & .15 & 7.8 \\
\hline & & 9 & $50-110$ & 52.4 & 27.3 & 20.3 & S clay loam & 11.4 & 3.5 & 7.80 & 15.7 & 0.22 & 0.13 & 12.0 \\
\hline & & & $110-150$ & 61.3 & 23.8 & 14.9 & Sandy loam & 13.6 & 3.9 & 7.70 & 12.9 & 0.24 & 0.14 & \begin{tabular}{|l|}
11.8 \\
\end{tabular} \\
\hline & & & WPM & 54.4 & 25.8 & 19.8 & S clay loam & 12.8 & 3.4 & 7.79 & 15.5 & 0.23 & 0.14 & 10.8 \\
\hline & & & $0-15$ & 74.9 & 15.5 & 9.6 & Sandy loam & 21.3 & 8.6 & 7.73 & 10.1 & 0.26 & 0.11 & 22.3 \\
\hline & & & $15-40$ & 69.7 & \begin{tabular}{|l|}
20.8 \\
\end{tabular} & 9.5 & Sandy loam & 15.3 & 7.0 & 7.77 & 10.0 & 0.17 & 0.15 & \begin{tabular}{|l|}
23.2 \\
\end{tabular} \\
\hline & & 10 & $40-70$ & 67.1 & 22.6 & 10.3 & Sandy loam & 17.5 & 6.9 & 7.76 & 10.4 & 0.19 & 0.16 & 22.3 \\
\hline & & & $70-110$ & 69.5 & 21.2 & 9.3 & Sandy loam & 16.8 & 8.5 & 7.79 & 9.9 & 0.16 & 0.14 & 22.7 \\
\hline & & & WPM & 69.6 & 20.7 & 9.7 & Sandy & 17.3 & 7.7 & 7.77 & 10.1 & 0.18 & 0.14 & 22.6 \\
\hline & & & & 63.7 & 23.0 & 13.3 & & 14.3 & 16.4 & 7.79 & 12.0 & 0.19 & 0.17 & 29.0 \\
\hline & $=$ & & $20-50$ & 70.3 & 13.5 & 16.2 & oam & 16.6 & 7.1 & \begin{tabular}{|l|}
7.77 \\
\end{tabular} & 13.6 & 0.26 & 0.11 & \begin{tabular}{|l|}
18.2 \\
\end{tabular} \\
\hline & & 1 & $50-120$ & 81.4 & 13.1 & 5.5 & Loamy sand & 11.7 & 6.3 & 7.76 & 7.9 & 0.24 & 0.13 & 17.6 \\
\hline & & & WPM & 75.7 & \begin{tabular}{|l|}
14.8 \\
\end{tabular} & 9.5 & Sandy loam & 13.4 & 8.2 & 7.76 & 10.0 & 0.24 & 0.13 & 19.7 \\
\hline & & & $0-25$ & 68.0 & 22.0 & 10.0 & Sandy loam & 14.9 & 10.2 & 7.78 & 10.3 & 0.22 & 0.16 & 22.6 \\
\hline & & & $25-50$ & 72.3 & 12.5 & 15.2 & Sandy loam & 16.7 & 7.4 & 7.76 & 13.0 & 0.28 & 0.08 & 19.1 \\
\hline & & 1 & $50-140$ & 67.5 & 22.2 & 10.3 & Sandy loam & 13.5 & 7.2 & 7.75 & 10.4 & 0.26 & 0.11 & 21.4 \\
\hline & & & WPM & 68.5 & 20.4 & 11.1 & Sandy loam & 14.3 & 7.8 & 7.75 & 10.9 & 0.26 & 0.11 & 21.2 \\
\hline
\end{tabular}

${ }^{1} \mathrm{P}$ No $=$ Profile No, ${ }^{2} \mathrm{WPM}=$ weighted profile mean, ${ }^{3} \mathrm{~S}$ clay loam $=$ Sandy clay loam, ${ }^{4} \mathrm{EC}=\mathrm{dSm}^{-1}$,

${ }^{5} \mathrm{pH}$ in $1: 2.5,{ }^{6} \mathrm{CEC}=\mathrm{Cmole} / \mathrm{Kg},{ }^{7} \mathrm{Gyp}=$ Gypsum 
Table (3): Cont.

\begin{tabular}{|c|c|c|c|c|c|c|c|c|c|c|c|c|c|c|}
\hline \multicolumn{2}{|c|}{ Landform } & \multirow{2}{*}{$\begin{array}{c}P \\
\text { No. }\end{array}$} & \multirow{2}{*}{$\begin{array}{l}\text { Depth } \\
\text { cm }\end{array}$} & \multicolumn{3}{|c|}{$\begin{array}{c}\text { Particle size } \\
\text { distribution \% }\end{array}$} & \multirow{2}{*}{$\begin{array}{c}\text { Texture } \\
\text { Class }\end{array}$} & \multirow{2}{*}{$\begin{array}{c}\mathrm{CaCO}_{3} \\
\%\end{array}$} & \multirow[t]{2}{*}{ EC } & \multirow[t]{2}{*}{$\mathrm{pH}$} & \multirow[t]{2}{*}{ CEC } & \multirow{2}{*}{$\begin{array}{c}\text { Gyp } \\
\%\end{array}$} & \multirow{2}{*}{$\begin{array}{c}\text { O M } \\
\%\end{array}$} & \multirow{2}{*}{$\begin{array}{c}\text { ESP } \\
\%\end{array}$} \\
\hline \multirow{16}{*}{ 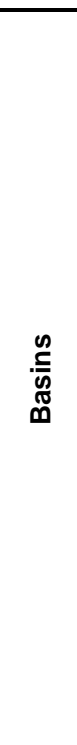 } & \multirow{16}{*}{ 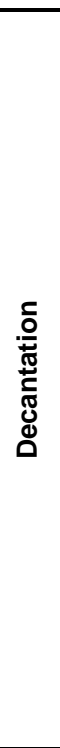 } & & & & & & & & & & & & & \\
\hline & & \multirow{5}{*}{13} & $0-25$ & 41.5 & 31.0 & 27.5 & Clay loam & 9.3 & 3.50 & 7.77 & 19.5 & 0.26 & 0.17 & 13.1 \\
\hline & & & $25-65$ & 22.4 & 36.9 & 40.7 & Clay & 3.5 & 2.71 & 7.81 & 26.5 & 0.07 & 0.11 & 10.2 \\
\hline & & & $65-110$ & 86.8 & 10.2 & 3.0 & Sand & 7.8 & 2.30 & 7.77 & 6.6 & 0.14 & 0.27 & 9.7 \\
\hline & & & $110-150$ & 26.5 & 37.8 & 35.7 & Clay loam & 4.7 & 2.00 & 7.81 & 23.9 & 0.14 & 0.29 & 10.7 \\
\hline & & & WPM & 46.0 & 28.1 & 25.9 & Loam & 6.07 & 2.52 & 7.80 & 10.7 & 0.14 & 0.22 & 10.7 \\
\hline & & \multirow{5}{*}{14} & $0-30$ & 64.4 & 27.5 & 8.1 & Sandy loam & 11.2 & 3.44 & 7.78 & 9.3 & 0.22 & 0.18 & 8.3 \\
\hline & & & $30-60$ & 27.5 & 36.3 & 36.2 & Clay loam & 4.7 & 3.53 & 7.73 & 24.2 & 0.15 & 0.23 & 10.0 \\
\hline & & & $60-110$ & 23.9 & 37.7 & 38.4 & Clay loam & 5.5 & 4.14 & 7.82 & 25.3 & 0.14 & 0.25 & 11.0 \\
\hline & & & $110-150$ & 24.2 & 38.5 & 37.3 & Clay loam & 6.5 & 3.63 & 7.81 & 24.7 & 0.13 & 0.29 & 11.1 \\
\hline & & & WPM & 32.8 & 35.6 & 31.6 & Clay loam & 6.74 & 3.74 & 7.79 & 21.7 & 0.16 & 0.24 & 10.30 \\
\hline & & \multirow{5}{*}{15} & $0-20$ & 62.7 & 27.2 & 10.1 & Sandy loam & 12.3 & 3.21 & \begin{tabular}{|l|}
7.72 \\
\end{tabular} & 10.3 & 0.23 & 0.17 & 9.0 \\
\hline & & & $20-50$ & 25.7 & 37.5 & 36.8 & Clay loam & 5.3 & 3.00 & 7.71 & 24.5 & 0.15 & 0.24 & 9.4 \\
\hline & & & $50-120$ & 24.8 & 36.7 & 38.5 & $\mathrm{am}$ & 6.5 & 4.20 & 7.81 & 25.4 & 0.13 & 0.26 & 10.7 \\
\hline & & & $120-150$ & 22.2 & 40.5 & 37.3 & Viay inc & 5 & 3.50 & 7.79 & 24.7 & 0.15 & 0.31 & 10.6 \\
\hline & & & WPM & 29.5 & 36.4 & 34.1 & Clay loam & 6.83 & 3.68 & 7.77 & 23.6 & 0.15 & 0.25 & 10.17 \\
\hline \multirow{27}{*}{ 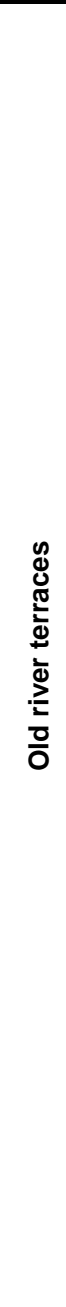 } & \multirow{13}{*}{ 矛 } & & $0-20$ & 24.9 & 37.5 & 37.6 & Clay loam & 4.5 & 4.31 & 7.77 & 24.9 & \begin{tabular}{|l|}
0.18 \\
\end{tabular} & 0.23 & 13.6 \\
\hline & & & $20-60$ & 29.2 & 36.2 & 34.6 & Clay loam & 4.2 & 8.50 & 7.78 & 23.3 & 0.13 & 0.28 & 24.7 \\
\hline & & 16 & $60-115$ & 28.9 & 36.8 & 34.3 & $\bar{m}$ & 5.3 & 7.50 & 7.79 & 23.1 & 0.15 & 0.25 & 22.2 \\
\hline & & & -150 & 29.7 & 3 & 35.0 & Giay & 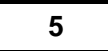 & 9.50 & 7.89 & 23.5 & 0.19 & .29 & 22.9 \\
\hline & & & WPM & 28.6 & 36.4 & 35.0 & Clay loam & 4.83 & 7.80 & 7.80 & 23.5 & 0.16 & 0.26 & 21.90 \\
\hline & & & $0-15$ & 21.3 & 39.4 & 39.3 & Clay loam & 12.3 & 5.91 & 7.88 & 25.8 & 0.14 & 0.27 & 13.6 \\
\hline & & & $15-80$ & 21.7 & 37.7 & 40.6 & Clay & 5.3 & 5.32 & 7.86 & 26.5 & 0.08 & 0.31 & 12.6 \\
\hline & & 16 & $80-150$ & 22.5 & 38.0 & 39.5 & Clay loan & 6 & 3.80 & 7.81 & 25.9 & 0.12 & 0.28 & 11.0 \\
\hline & & & WPM & 22.0 & 38.0 & 40.0 & $\bar{C}$ & 0.00 & 4.66 & 7.84 & 26.2 & 0.1 & 0.29 & 11.9 \\
\hline & & & $0-20$ & 23.4 & 36.4 & 40.2 & Clay & 14.1 & 5.13 & 7.80 & 26.3 & 0.16 & 0.29 & 12.7 \\
\hline & & & $20-90$ & 23.4 & 37.1 & 39.5 & Clay loam & 3.5 & 4.21 & 7.70 & 25.9 & 0.09 & 0.33 & 9.9 \\
\hline & & 18 & $90-150$ & 23.3 & 38.3 & 38.4 & Clay loam & 4.2 & 3.90 & 7.70 & 25.3 & 0.14 & 0.26 & 11.8 \\
\hline & & & WPM & 23.4 & 37.5 & 39.1 & Clay loam & 5.19 & 4.20 & 7.71 & 25.7 & 0.12 & 0.30 & 11.0 \\
\hline & & & $0-20$ & 82.2 & 13.0 & 4.8 & Luainy santa & 14 & 8.72 & 7.77 & 7.5 & 0.29 & 0.11 & 25.1 \\
\hline & & 19 & $20-55$ & 81.3 & 13.2 & 5.5 & Loamy sand & 7.3 & 12.51 & 7.79 & 7.9 & 0.23 & 0.13 & 28.5 \\
\hline & & 19 & $55-100$ & 30.5 & 35.2 & 34.3 & Clay loam & 15.2 & 8.30 & 7.79 & 23.1 & 0.13 & 0.21 & 24.1 \\
\hline & & & WPM & 58.6 & 23.1 & 18.3 & Sandy loam & 12.2 & 9.85 & 7.78 & 14.7 & 0.2 & 0.16 & 25.8 \\
\hline & & & $0-25$ & 25.6 & 38.0 & 36.4 & Clay loam & 5.5 & 2.93 & 7.78 & 24.3 & 0.13 & 0.25 & 6.5 \\
\hline & 흘 & & $25-60$ & 23.2 & 39.3 & 37.5 & Clay loam & 4.9 & 2.21 & 7.75 & 24.8 & 0.14 & 0.27 & 8.1 \\
\hline & $\frac{\lambda}{0}$ & 20 & $60-100$ & 30.4 & 35 & 34.6 & Clay loam & 5.2 & 3.30 & 7.79 & 23.3 & 0.17 & 0.24 & 16.6 \\
\hline & $\frac{\pi}{0}$ & & $100-150$ & 27.4 & 37.6 & 35.0 & Clay loam & 5.6 & 4.00 & 7.77 & 23.5 & 0.16 & 0.21 & 12.5 \\
\hline & 웅 & & WPM & 26.9 & 37.4 & 35.7 & Clay loam & 5.31 & 3.21 & 7.77 & 23.9 & 0.15 & \begin{tabular}{|l|}
0.24 \\
\end{tabular} & 11.5 \\
\hline & & & $0-20$ & 30.9 & 39.2 & 29.9 & Clay loam & 6.2 & 3.73 & 7.81 & 20.8 & 0.14 & 0.22 & 11.6 \\
\hline & & & $20-50$ & 30.2 & 36.6 & 33.2 & Clay loam & 7.5 & 3.42 & 7.78 & 22.6 & 0.13 & \begin{tabular}{|l|}
0.24 \\
\end{tabular} & 8.3 \\
\hline & & 21 & $50-110$ & 27.0 & 38.3 & 34.7 & Clay loam & 5.5 & 2.70 & \begin{tabular}{|l|}
7.75 \\
\end{tabular} & 23.4 & 0.16 & \begin{tabular}{|l|}
0.21 \\
\end{tabular} & 10.8 \\
\hline & & & $110-150$ & 29.2 & 36.3 & 34.5 & Clay loam & 4.5 & 3.10 & \begin{tabular}{|l|}
7.74 \\
\end{tabular} & 23.3 & 0.14 & 0.23 & 10.4 \\
\hline & & & WPM & 28.7 & 37.6 & 33.7 & Clay loam & 5.73 & 3.08 & 7.76 & 22.8 & 0.15 & 0.22 & 10.3 \\
\hline
\end{tabular}


M.S. Amira, er al.,

Table (3): Cont.

\begin{tabular}{|c|c|c|c|c|c|c|c|c|c|c|c|c|c|c|}
\hline \multirow{2}{*}{\multicolumn{2}{|c|}{ Landform }} & \multirow{2}{*}{$\begin{array}{c}P \\
\text { No }\end{array}$} & \multirow{2}{*}{$\begin{array}{l}\text { Depth } \\
\text { cm }\end{array}$} & \multicolumn{3}{|c|}{$\begin{array}{c}\text { Particle size } \\
\text { distribution \% }\end{array}$} & \multirow{2}{*}{$\begin{array}{l}\text { Texture } \\
\text { Class }\end{array}$} & \multirow{2}{*}{$\begin{array}{c}\mathrm{CaCO}_{3} \\
\%\end{array}$} & \multirow[t]{2}{*}{ EC } & \multirow[t]{2}{*}{$\mathrm{pH}$} & \multirow[t]{2}{*}{ CEC } & \multirow{2}{*}{$\begin{array}{c}\text { Gyp } \\
\%\end{array}$} & \multirow{2}{*}{$\begin{array}{c}\text { O. M } \\
\%\end{array}$} & \multirow{2}{*}{$\begin{array}{c}\text { ESP } \\
\%\end{array}$} \\
\hline & & & & Sand & Silt & Clay & & & & & & & & \\
\hline \multirow{27}{*}{ 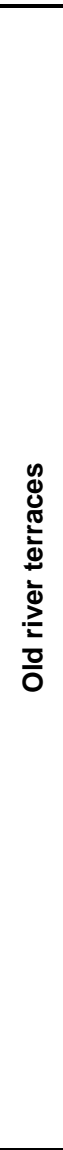 } & \multirow{14}{*}{$\begin{array}{l}\frac{0}{\pi} \\
\frac{\pi}{0} \\
\text { 0 } \\
\Sigma\end{array}$} & \multirow{4}{*}{22} & $0-25$ & 30.4 & 35.0 & 34.6 & Clay loam & 5.3 & 5.3 & 7.78 & 23.3 & 0.15 & 0.25 & 14.2 \\
\hline & & & $25-70$ & 31.2 & 35.3 & 33.5 & Clay loam & 5.0 & 3.5 & 7.77 & 22.7 & 0.11 & 0.23 & 12.2 \\
\hline & & & $70-130$ & 23.9 & 38.6 & 37.5 & Clay loam & 4.8 & 2.5 & 7.76 & 24.8 & 0.13 & 0.27 & 8.6 \\
\hline & & & WPM & 27.7 & 36.8 & 35.5 & Clay loam & 5.0 & 3.4 & 7.77 & 23.8 & 0.13 & 0.25 & 10.9 \\
\hline & & \multirow{5}{*}{23} & $0-30$ & 29.6 & 35.3 & 35.1 & Clay loam & 5.5 & 2.8 & 7.79 & 23.6 & 0.13 & 0.26 & 7.2 \\
\hline & & & $30-50$ & 27.2 & 36.4 & 36.4 & Clay loam & 4.6 & 3.6 & 7.84 & 24.3 & 0.15 & 0.24 & 10.6 \\
\hline & & & \begin{tabular}{|l|}
$50-100$ \\
\end{tabular} & 28.4 & 35.9 & 35.7 & Clay loam & 4.8 & 2.6 & 7.75 & 23.9 & 0.13 & 0.23 & 10.3 \\
\hline & & & \begin{tabular}{|l|}
$100-150$ \\
\end{tabular} & 38.2 & 31.3 & 30.5 & Clay loam & 6.7 & 2.7 & 7.77 & 21.1 & 0.27 & 0.15 & 10.6 \\
\hline & & & WPM & 31.8 & 34.3 & 33.9 & Clay loam & 5.5 & 2.8 & 7.78 & 23.0 & 0.18 & 0.21 & 9.8 \\
\hline & & \multirow{5}{*}{24} & $0-30$ & 31.0 & 34.7 & 34.3 & Clay loam & 4.5 & 3.2 & 7.79 & 23.1 & 0.15 & 0.23 & 8.4 \\
\hline & & & $30-60$ & 27.6 & 37.1 & 35.3 & Clay loam & 6.6 & 3.5 & 7.78 & 23.7 & 0.13 & 0.24 & 10.9 \\
\hline & & & \begin{tabular}{|l|}
$60-120$ \\
\end{tabular} & 27.0 & 36.6 & 36.4 & Clay loam & 7.5 & 2.5 & 7.72 & 24.3 & 0.12 & 0.22 & 9.7 \\
\hline & & & \begin{tabular}{|l|}
$120-150$ \\
\end{tabular} & 38.2 & 32.3 & 29.5 & Clay loam & 9.7 & 2.9 & 7.79 & 20.6 & 0.23 & 0.13 & 9.5 \\
\hline & & & WPM & 30.1 & 35.5 & 34.4 & Clay loam & 7.2 & 2.9 & 7.76 & 23.2 & .15 & 0.21 & 9.6 \\
\hline & \multirow{13}{*}{ 30 } & \multirow{5}{*}{25} & $0-10$ & 23.2 & 35.0 & 41.8 & Clay & 5 & 3.0 & 7.78 & 27.1 & 0.15 & 0.25 & 8.6 \\
\hline & & & 10--40 & 22.2 & 37.2 & 40.6 & Clay & 3.5 & 2.9 & 7.77 & 26.5 & 0.12 & 0.27 & 9.8 \\
\hline & & & \begin{tabular}{|l|}
$40-110$ \\
\end{tabular} & 21.9 & 36.8 & 41.3 & Clay & 4.2 & 3.6 & 7.79 & 26.9 & 0.11 & 0.29 & 13.3 \\
\hline & & & \begin{tabular}{|l|}
$110-150$ \\
\end{tabular} & 23.2 & 38.5 & 38.3 & Clay loam & 3.5 & 3.5 & 7.78 & 25.3 & 0.13 & 0.28 & 15.2 \\
\hline & & & WPM & 22.4 & 37.2 & 40.4 & Clay & 3.9 & 3.4 & 7.78 & 26.4 & 0.12 & 0.28 & 12.8 \\
\hline & & \multirow{3}{*}{26} & $0-25$ & 22.1 & 36.8 & 41.1 & Clay & 7.8 & 3.6 & 7.79 & 26.8 & 0.11 & 0.23 & 11.8 \\
\hline & & & $25-70$ & 32.5 & 34.0 & 33.5 & Clay loam & 6.6 & 5.4 & 7.87 & 22.7 & 0.12 & 0.22 & 15.3 \\
\hline & & & WPM & 28.8 & 35.0 & 36.2 & Clay loam & 7.0 & 4.7 & 7.84 & 24.2 & 0.12 & 0.22 & 14.0 \\
\hline & & & $0-20$ & 25.3 & 33.1 & 41.6 & Clay & 7.9 & 3.2 & 7.76 & 27.0 & 0.15 & 0.26 & 12.4 \\
\hline & & & $20--40$ & 30.0 & 34.7 & 35.3 & Cla & 4.3 & 2.8 & 7.78 & 23.7 & 0.11 & 0.22 & 10.0 \\
\hline & & 27 & \begin{tabular}{|l|}
$40-115$ \\
\end{tabular} & 23.4 & 39.9 & 36.7 & Clay loam & 6.6 & 3.7 & 7.80 & 24.4 & 0.14 & 0.13 & 12.6 \\
\hline & & & \begin{tabular}{|l|}
$115-150$ \\
\end{tabular} & 31.3 & 36.2 & 32.5 & Clay loam & 6.5 & 5.5 & 7.85 & 22.2 & 0.11 & 0.24 & 14.9 \\
\hline & & & WPM & 26.4 & 37.4 & 36.2 & Clay loam & 6.4 & 3.9 & 7.80 & 24.1 & 0.13 & 0.18 & 12.7 \\
\hline & & & 10 & 82.2 & 12.5 & 5.3 & Loamy sand & 8. & 12.3 & 7.93 & 7.8 & .28 & 0.07 & 24.2 \\
\hline & & & $10--40$ & 86.4 & 11.0 & 2.6 & 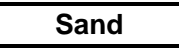 & 1.0 & 10.6 & 7.85 & 6.3 & 0.27 & 0.07 & 26.1 \\
\hline & & 2 & \begin{tabular}{|l|}
$40-100$ \\
\end{tabular} & 23.4 & 38.9 & 37.7 & Clay loam & 4.4 & 7.5 & 7.87 & 25.0 & 0.15 & 0.29 & 23.2 \\
\hline & & & 100-150 & 30.2 & 35.5 & 34.3 & Clay loam & 4.5 & 8.4 & 7.89 & 23.1 & 0.14 & 0.23 & 18.5 \\
\hline & & & WPM & 42.2 & 30.4 & 27.4 & Clay loam & 5.2 & 8.7 & 7.88 & 19.5 & 0.18 & 0.21 & 22.3 \\
\hline 0 & 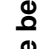 & & $0-15$ & 80.2 & 15.5 & 4.3 & Loamy sand & 6.9 & 13.3 & 7.98 & 7.2 & 0.26 & 0.06 & 22.1 \\
\hline$\frac{a}{2}$ & $\frac{\mathscr{d}}{\underline{\underline{J}}}$ & & 15--50 & 86.4 & 11.0 & 2.6 & Sand & 7.7 & 11.3 & 7.88 & 6.3 & 0.25 & 0.04 & 25.2 \\
\hline $\bar{\Phi}$ & ¿ & 29 & $50-110$ & 25.6 & 37.9 & 36.5 & Clay loam & 4.8 & 8.5 & 7.89 & 24.3 & 0.14 & 0.26 & 22.8 \\
\hline$\sigma_{2}$ & $=$ & & \begin{tabular}{|l|}
$110-150$ \\
\end{tabular} & 30.2 & 35.7 & 34.1 & Clay loam & 6.5 & 8.8 & 7.86 & 23.0 & 0.12 & 0.24 & 16.4 \\
\hline & & & WPM & 46.5 & 28.8 & 24.7 & Loam & 6.1 & 9.7 & 7.89 & 18.1 & 0.17 & 0.18 & 21.6 \\
\hline & & & $0-30$ & 82.2 & 11.2 & 6.6 & Loamy sand & 7.4 & 11.5 & 7.88 & 8.5 & 0.29 & 0.11 & 23.0 \\
\hline & & & $30--80$ & 85.4 & 11.4 & 3.2 & Loamy sand & 6.9 & 12.0 & 7.87 & 6.7 & 0.24 & 0.09 & 26.2 \\
\hline & & 30 & \begin{tabular}{|l|}
$80-100$ \\
\end{tabular} & 25.4 & 38.9 & 35.7 & Clay loam & 5.1 & 8.5 & 7.87 & 23.9 & 0.17 & 0.28 & 24.4 \\
\hline & & & \begin{tabular}{|l|}
$100-150$ \\
\end{tabular} & 27.2 & 36.6 & 36.2 & Clay loam & 5.2 & 8.1 & 7.84 & 24.2 & 0.14 & 0.21 & 18.4 \\
\hline & & & WPM & 57.4 & 23.4 & 19.2 & Sandy loam & 6.2 & 10.1 & 7.86 & 15.1 & 0.21 & 0.16 & 22.7 \\
\hline
\end{tabular}

\section{Soil classification}

Based on the climatic condition, soil morphological and physiochemical characteristics, the studied soils are classified up to sub great group level according to Soil Survey Staff (2014). According to FAO (1977) and USDANRCS (1997), the dominant soil moisture 
regime of this area is "Torric" with "Thermic" soil temperature regime. Most of these soils haven't any clear diagnostic sub-surface horizons and therefore are classified under Entisols order (Table, 4). About $50.77 \%$ from the studied soils have $>30$ swelling clay contents and cracked when not irrigated (Tables, 3 and 4), therefore they classified as Vertic Torriorthents. The soils of profiles 5,8 and 9 have light loamy texture and classified as Typic Torriorthents (18.61\%). The soils of profiles $11,12,16,19,28,29$ and 30 have a clear sodicity effect on the hall profile layers (Tables 3 and 4 ) and classified as Sodic Torriorthents (14.27). The soils of profile 26 have a highwater table level (70 $\mathrm{cm}$ from the soil surface) and classified as Aquic Torriorthents (0.95). Only, the soils of profile 10 in the Overflow basin have both Calcic and Sodic horizons and classified under Aridisols as Sodic Haplocalcids. Classification of the studied soils are presented in Table (4) and Fig (4).

Table (4): Soil classification in the study area.

\begin{tabular}{|c|c|c|c|c|}
\hline \multirow{2}{*}{ Order } & \multirow{2}{*}{ Sub Great Group } & \multirow{2}{*}{ Soils of profiles No. } & \multicolumn{2}{|c|}{ Area } \\
\hline & & & $\mathrm{Km}^{2}$ & $\%$ \\
\hline \multirow{3}{*}{ Entisols } & Vertic Torriorthents & $\begin{array}{l}1,2,3,4,6,7,13,14,15,17 \\
18,20,21,22,23,24,25,27\end{array}$ & 1085.8 & 50.77 \\
\hline & Typic Torriorthents & $5,8,9$ & 397.9 & 18.61 \\
\hline & Sodic Torriorthents & $11,12,16,19,28,29,30$ & 305.1 & 14.27 \\
\hline & Aquic Torriorthents & 26 & 20.4 & 0.95 \\
\hline Aridisols & Sodic Haplocalcids & 10 & 86.96 & 4.07 \\
\hline \multicolumn{3}{|c|}{ Water bodies } & 242.35 & 11.33 \\
\hline \multicolumn{3}{|c|}{ Total } & 2138.53 & 100 \\
\hline
\end{tabular}

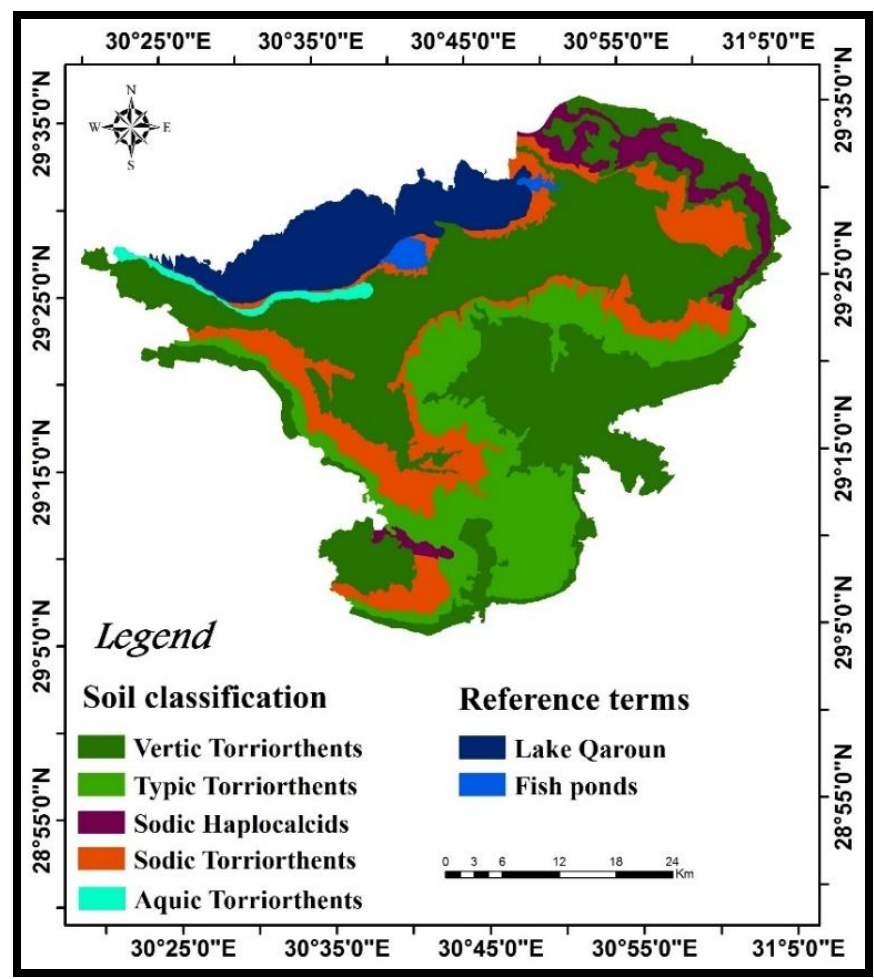

Fig (4): Soil classification map. 


\section{REFERANCES}

Burt, Rebecca and Soil Survey Staff (2014). Kellogg Soil Survey Laboratory Methods Manual, Soil Survey Investigations Report No. 42, Version 5.0, Kellogg Soil Survey Laboratory, National Soil Survey Center, Natural Resources Conservation Service, USDA, Lincoln, Nebraska, USA.

Dawoud, M.A., M.M. Darwish and M.M. ElKady (2005). GIS-based groundwater management model for Western Nile Delta. Water Resources Manag. 19: 585-604.

Dobos, E., B. Norman, W. Bruee, M. Luca, J. Chris and M. Erika (2002). The use of DEM and satellite images for regional scale soil database. Proceedings of the 17th World Congress of Soil Science, Bangkok.

ESRI "Environmental Systems Research Institute" (2003). Using ArcGIS Geostatistical Analyst. Environmental Systems Research Institute (ESRI) Press, Redlands, California.

ESRI "Environmental Systems Research Institute" (2014). Arc Map Version 10.1 User Manual. ESRI, 380 New York Street, Redlands, California, 923738100, USA.

FAO (1977). Soil map of the world 1 : 5 000 000, Volume VI Africa, FAO, UNSCO, Paris.

FAO (2006). Guidelines for soil profile description. Soil Res. Dev. and Co. Serv., Land and Water Dev. Div., Rome, Italy.
Hegazi, A.M., M.Y. Afifi, M.A. EL Shorbagy, A.A. Elwan and S. ElDemerdashe (2005). Egyptian national action program to combat desertification, Arab Republic of Egypt, Ministry of Agriculture and Land Reclamation, Desert Research Center (DRC), UNCCD Egypt Office, 1 Mathaf EI Mataria - P.O.Box: 11753 EI Mataria, Cairo, Egypt.

ITT "International Telephone and Telegraph" (2017). ENVI 5.5 software help manual, ITT corporation, 1133 Westchester Avenue, White Plains, NY 10604, USA.

Lillesand, T. M. and R. W. Kiefer (2007). Remote Sensing and Image Interpretation. 5th Ed. Paper back. John Wiley, New York.

Soil Science Division Staff (2017). Soil Survey Manual, Handbook No. 1 USDA. 1400 Independence Avenue, SW, Washington, D.C. USA

Soil Survey Staff (2014). Keys to Soil Taxonomy, $11^{\text {th }}$ Ed., USDA, NRCS, Pocahontas Press, Inc., Blacksburg, Virginia, USA.

USDA-NRCS (1997). Soil climate map, Soil Science Division, World Soil Resources, United States Department of Agriculture \& Natural Recourses Conservation Service, Washington D.C.

Zinck, J.A. and C.R. Valenzuela (1990). Soil Geographic Database: Structure and Application Examples. ITC journal, 3: 270. 
دراسة حديثة عن جيومورفولوجيا وخصائص وتقسيم أراضي محافظة الفيوم، مصر

محمد سمير عراقي عميرة (1)، علاء الدين حسن النهري (2)، فوزي الثاذلي أبو عجوة (1)،

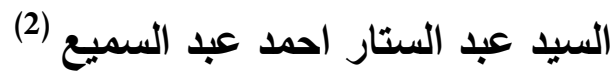

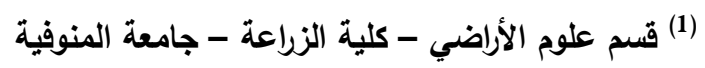

(2) الهيئة القومية للاستشعار من البعد وعلوم الفضاء - النقاهرة

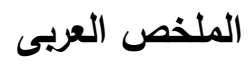

أجري هذا البحث بهاف اجراء دراسة حديثة عن الخصائص الجيومرفولوجية والفيزيوكيميائية وكنلك تقسيم أراضي

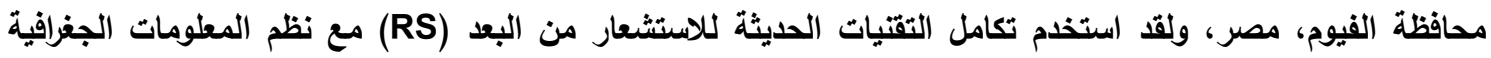
(GIS)

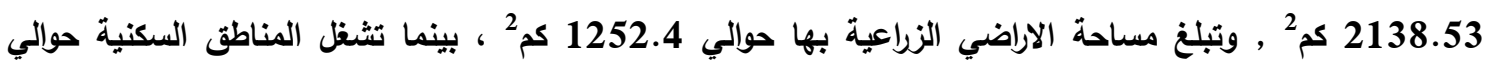

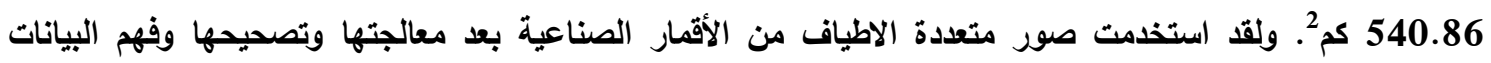

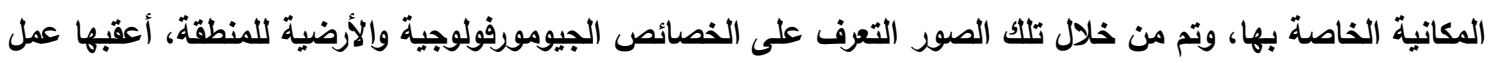
حصر نصف تفصيلي لها لتأكيد معلومات الخرائط الناتجة من الأمّار الصناعية،

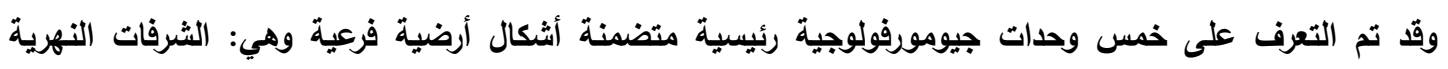

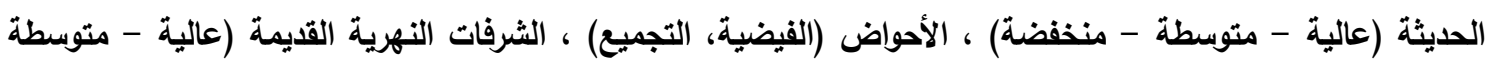

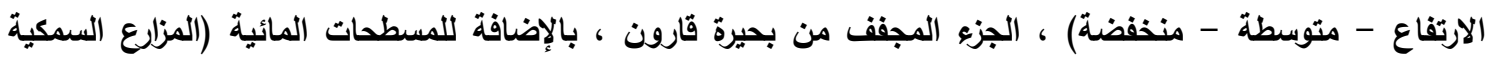

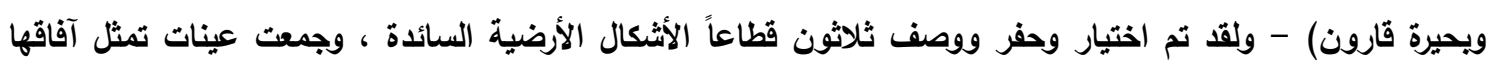
لتحليل خصائصها الطبيعية و الكيماوية. أوضحت الدراسات الحقلية أن سطح أراضي معظم الوحدات شبه مستوية إلى مائلة، ومعظم الأراضي عميقة القطاع،

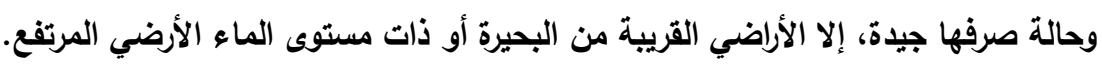

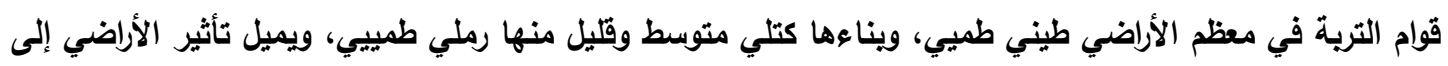

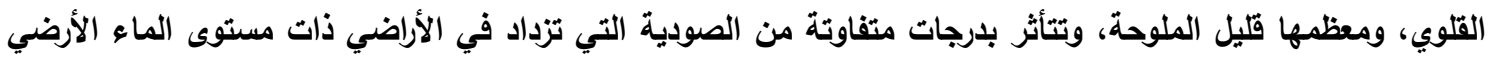

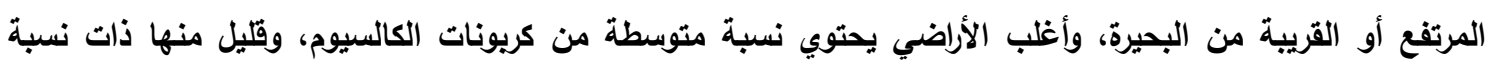

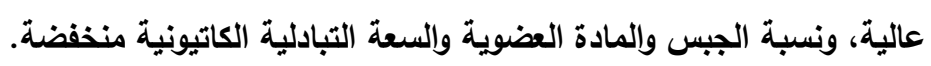

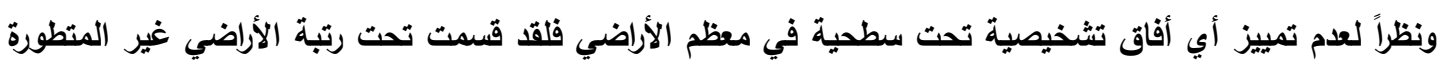
Entisols

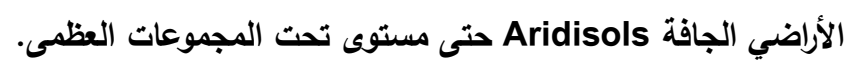
الكلمات الدالة: الاستشعار من البعد، نظم المعلومات الجغرافية، الوحدات الجيومورفولوجية، الخصائص الفيزيويوكيميائية كلأراضي، تقسيم الأراضي.

أسماء السادة المحكمين

كلية الزراعة - جامعة المنوفية

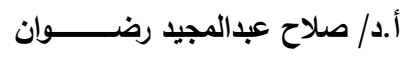

كلية الززاعة - جامعة المنوفية - جامعة المنية

أ.د/ الحسينى عبدالغفار أبو حسين 